\title{
Optimization of endoloop closure for gastric endoscopic submucosal dissection defects with a bead: the bead, loop, and clips technique
}

Endoscopic closure of endoscopic submucosal dissection (ESD)-induced gastric defects using an endoloop and endoclips may prevent delayed bleeding in patients who are receiving anticoagulants [1]. Several technical variations of the endoloop and endoclips technique have been described, some of which also use single-channel endoscopes [2], which are in widespread use. Their successful application is however being undermined by a number of pitfalls, both procedure-related (e.g. respiratory fluctuations, poor endoloop device maneuverability when kept outside the endoscope) and instrument-related (e.g. difficulty grasping the tail or stem of the endoloop, resulting from the hooked shape of the device) [3]. Herein, we illustrate a modification of the endoloop and endoclips technique, inspired by the "loop 9" method [4], that can be applied as a less cumbersome yet effective alternative to similar procedures.

The modified procedure, which we have termed "bead, loop, and clips (BLC) closure" was applied post-ESD for an early gastric cancer in a 68-year-old man who was receiving low dose aspirin. Initially, the stem of an endoloop is manually elongated so that it can be passed through a 6-mm bead. A 3-0 nylon thread is then tied onto the tail end of the endoloop, distally to the bead to prevent its migration ( $\mathbf{F i g} \mathbf{1} \mathbf{a}$ ). After the successful completion of the ESD, the "beaded" endoloop is passed through an overtube into the esophagus and stomach, and is anchored onto the edge of the mucosal defect using a reopenable endoclip. Additional endoclips are used to fix the endoloop to the margins of the defect.

A small caliber disposable biopsy forceps (Radial Jaw 4P, Boston Scientific, Boston, Massachusetts, USA) fitted into the outer

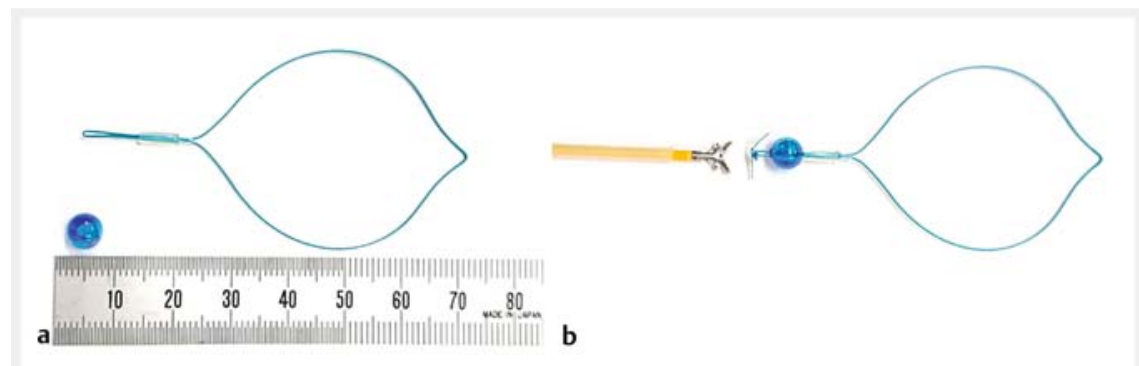

- Fig. 1 Photograph of the equipment used during the bead, loop, and clips technique, which includes: $\mathbf{a}$ an endoloop and a bead; $\mathbf{b}$ a biopsy forceps fitted into an outer sheath.

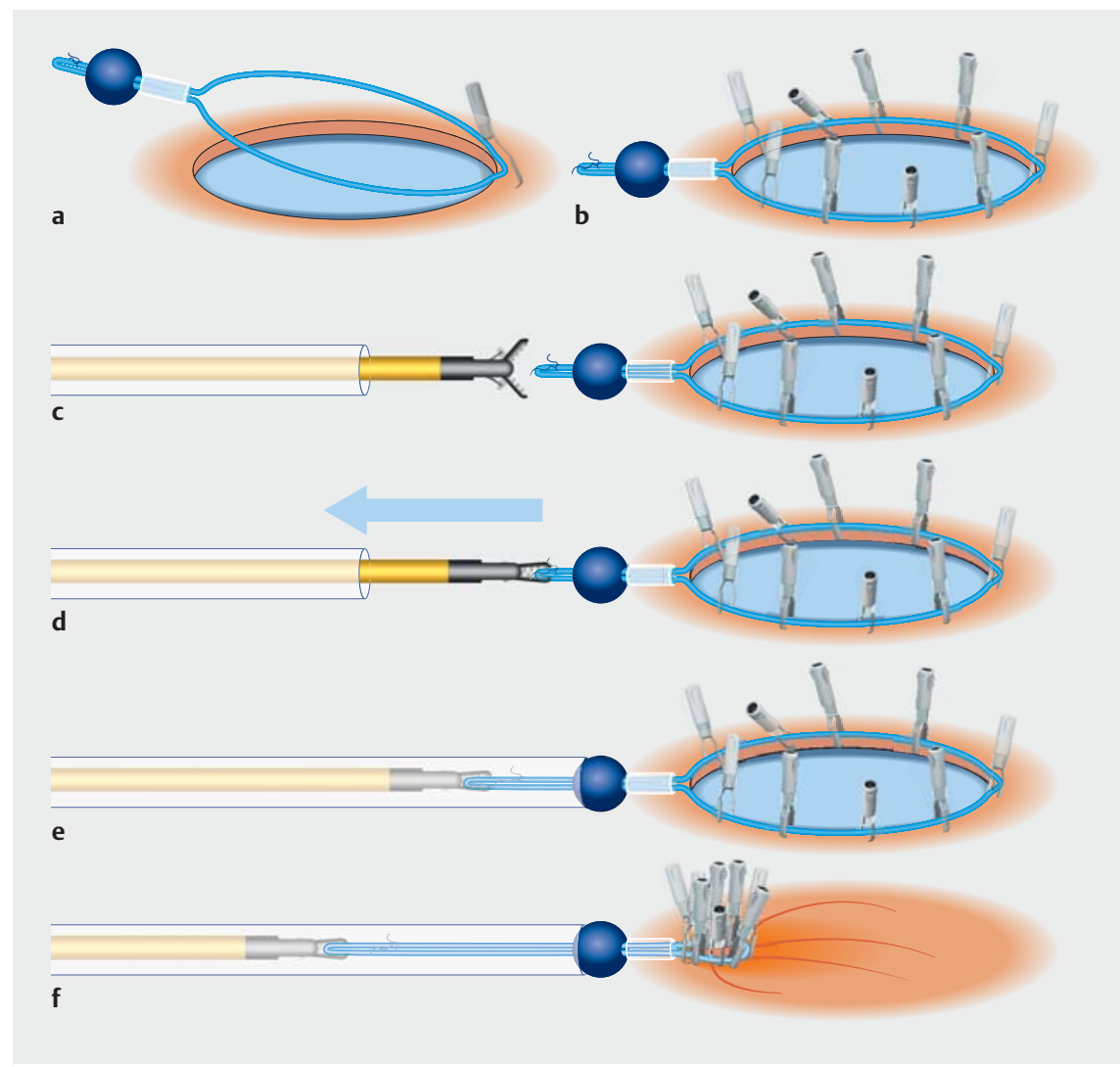

- Fig. 2 An illustration of the different steps during bead, loop, and clips (BLC) closure showing: $\mathbf{a}$ the endoloop with the bead being fixed to the edge of the mucosal defect; $\mathbf{b}$ the endoloop being fixed onto the margins of the defect with additional endoclips; $\mathbf{c}$ the endoloop tail being grasped by biopsy forceps fitted inside an outer sheath; $\mathbf{d}$ the endoloop being pulled into the sheath; e the bead serving as an "anchor" or "keeper"; $\mathbf{f}$ the endoloop being tightened to provide adequate defect closure. 

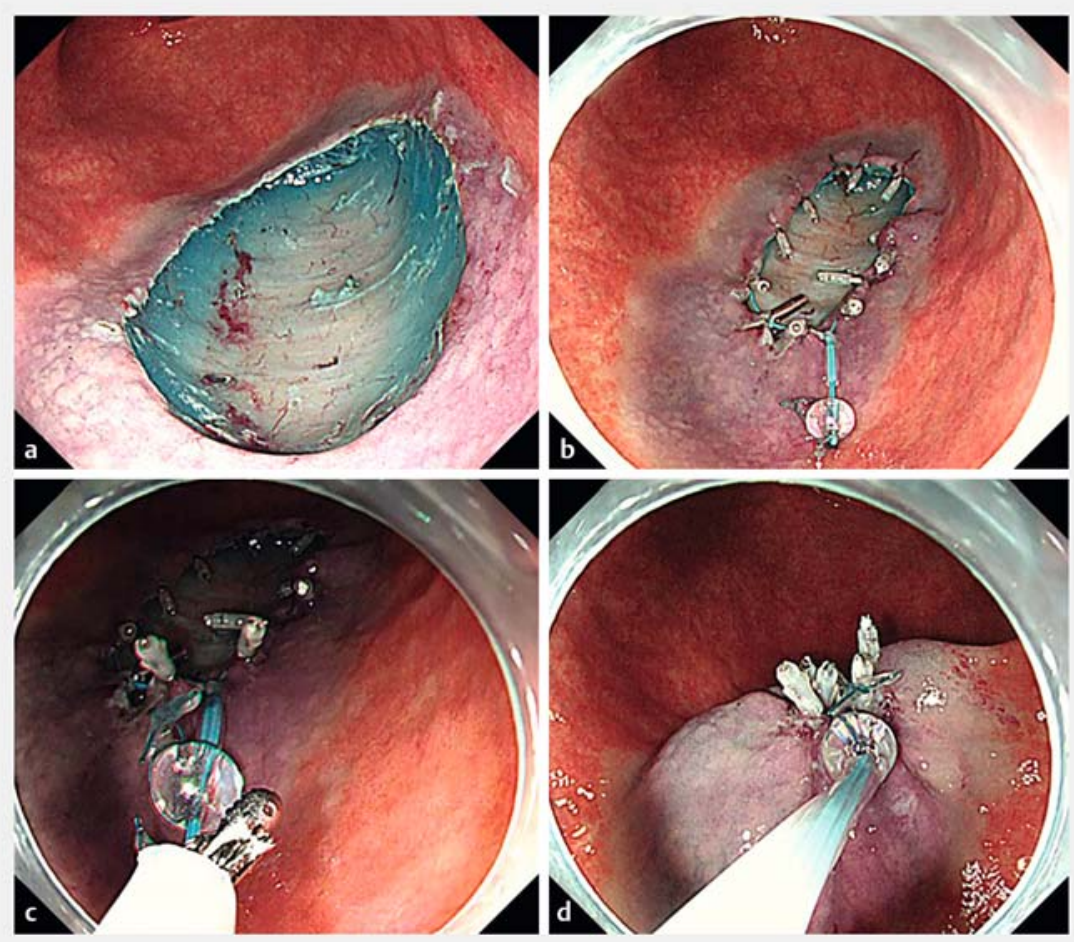

Fig. 3 Endoscopic views of the bead, loop, and clips (BLC) closure showing: a a mucosal defect left after endoscopic mucosal dissection; $\mathbf{b}$ the endoloop fixed with endoclips around the edge of the mucosal defect; $\boldsymbol{c}$ the endoloop tail being grasped by the biopsy forceps covered with the outer sheath; $\mathbf{d}$ the endoloop being tightened to provide adequate defect closure.

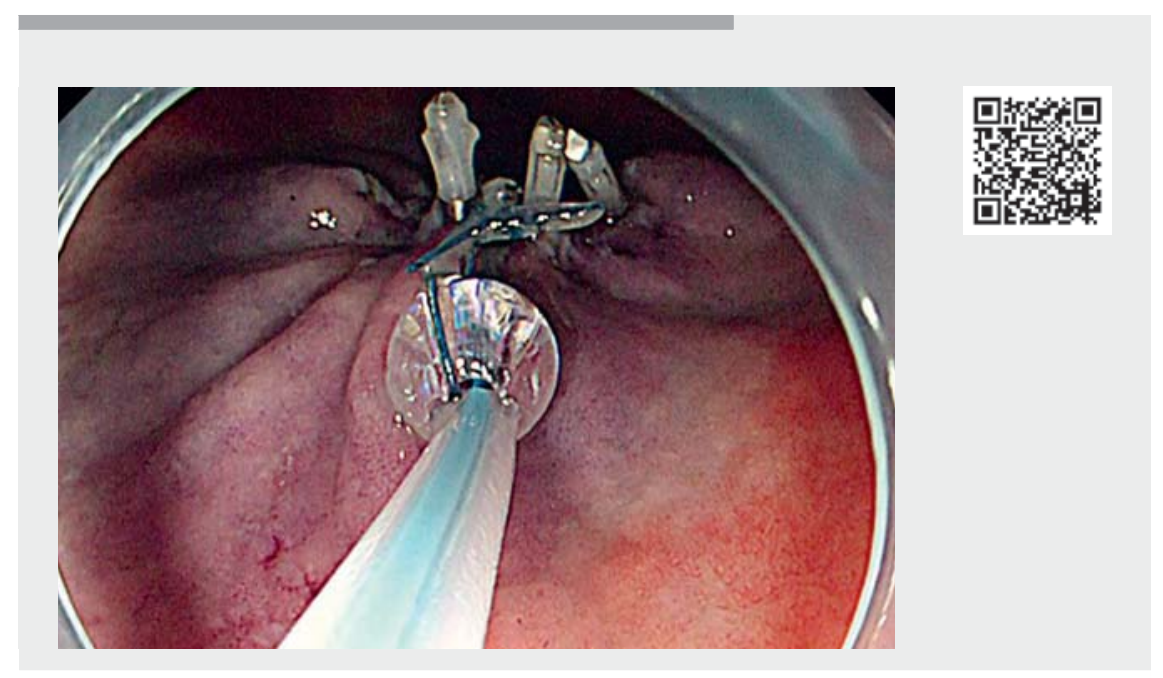

Video 1 Successful closure of an endoscopic submucosal dissection-induced gastric defect using the bead, loop, and clips (BLC) technique. sheath of a disposable endoloop device (HX-400U-30; Olympus, Tokyo, Japan) ( Fig.1 b) is then inserted through the single-channel endoscope to grasp the endoloop tail and pull it into the sheath. Throughout this process, the bead serves as an "anchor" or "keeper" that permits endoloop tightening and subsequent defect closure ( $\triangleright$ Fig.2). Finally, the bead can be removed using biopsy forceps. The entire procedure is shown in $>\mathbf{F i g . ~} \mathbf{3}$ and $>$ Video 1.

The procedure was uneventful and on endoscopy performed on the fourth day post-ESD, the defect remained closed (> Fig.4).

Endoscopy_UCTN_Code_TTT_1AO_2AN

\section{Competing interests}

The authors declare that they have no conflict of interest.

The authors

Yuka Kowazaki ${ }^{1}$, Anastassios Manolakis ${ }^{2,3}$, Hisashi Fukuda ${ }^{1,4}$, Itaru Saito', Tetsurou Miwata $^{1,4}$, Sawako Fujikura ${ }^{1,4}$, Takaaki Morikawa ${ }^{1,4}$

1 Department of Gastroenterology, Jyoban Hospital, Tokiwa Foundation, Fukushima, Japan

2 University of Thessaly, School of Medicine, Larissa, Greece

3 Department of Gastroenterology, University Hospital of Larissa, Larissa, Greece

4 Department of Medicine, Division of Gastroenterology, Jichi Medical University, Tochigi, Japan

Corresponding author

\section{Hisashi Fukuda, MD}

Department of Gastroenterology, Jyoban Hospital, Tokiwa Foundation, 57 Jyoban kamiyunagayamachi kaminodai, Iwaki, Fukushima, 972-8322, Japan r0849hf@jichi.ac.jp 


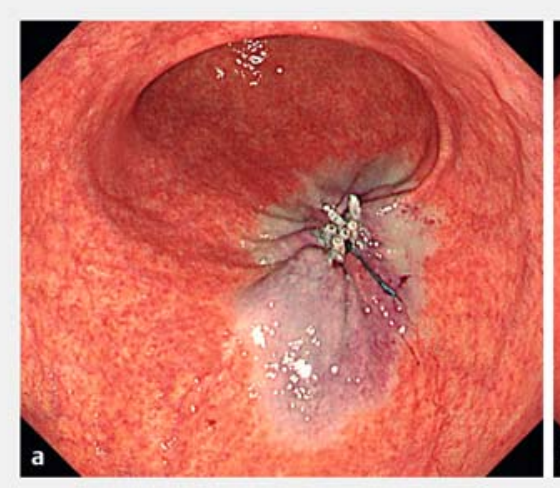

- Fig. 4 Endoscopic images of the defect: a immediately after defect closure; $\mathbf{b} 4$ days later, with the defect remaining completely closed.

\section{References}

[1] Shiotsuki K, Takizawa K, Notsu A et al. Endoloop closure following gastric endoscopic submucosal dissection to prevent delayed bleeding in patients receiving antithrombotic therapy. Scand J Gastroenterol 2021; 56: $1117-1125$

[2] Lua GW, Lie F. Closure of a large mucosal defect after endoscopic submucosal dissection using "pre-detached loop and clips" method with a single-channel gastroscope. Endoscopy 2015; 47: E464-E465

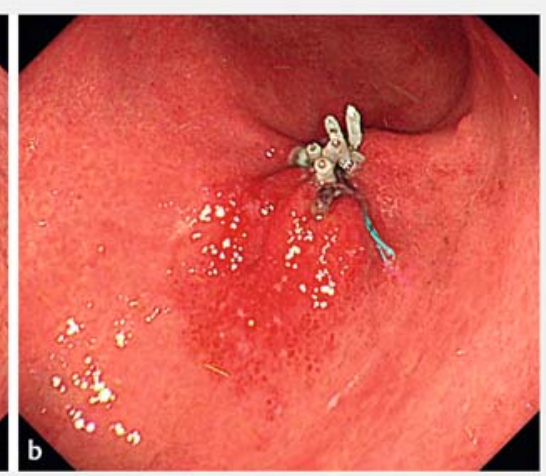

Bibliography

Endoscopy 2022; 54: E641-E643

DOI 10.1055/a-1731-7381

ISSN 0013-726X

published online 4.2.2022

(C) 2022. Thieme. All rights reserved.

Georg Thieme Verlag KG, Rüdigerstraße 14 , 70469 Stuttgart, Germany

\section{ENDOSCOPY E-VIDEOS}

https:/|eref.thieme.de/e-videos

口回回 Endoscopy E-Videos is an open access online section, 自䲝: reporting on interesting cases and new techniques in gastroenterological endoscopy. All papers include a high quality video and all contributions are freely accessible online. Processing charges apply (currently EUR 375), discounts and wavers acc. to HINARI are available.

[4] Inoue H, Tanabe M, Shimamura Y et al. A novel endoscopic purse-string suture technique, “loop 9", for gastrointestinal defect closure: a pilot study. Endoscopy 2021. doi:10.1055/a-1364-4160

This section has its own submission website at https://mc.manuscriptcentral.com/e-videos 